\title{
Profil bibliométrique et devenir des thèses soutenues à la faculté de médecine de Monastir (Tunisie)
}

\author{
Bibliometric profile and future of dissertations defended in the faculty of \\ medicine in Monastir (Tunisia)
}

\author{
Kamel Ben SAlEM ${ }^{1}$, Ines BouAnenE ${ }^{1}$, Nizar MosbahI ${ }^{1}$, Sana ElMHAMdi ${ }^{1}$, \\ Mohamed Soussi SolTANI ${ }^{1}$ et Ahmed Ben ABDELAZIZ ${ }^{2}$ \\ 1 Département de médecine communautaire, Faculté de médecine de Monastir, Monastir, Tunisie \\ 2 Direction des systèmes d'information, Centre hospitalier universitaire de Sahloul-Sousse, Sousse, Tunisie
}

Manuscrit reçu le 7 décembre 2010 ; commentaires éditoriaux formulés aux auteurs le 21 juillet 2011 ; accepté pour publication le 27 juillet 2011

\begin{abstract}
Mots clés :
Bibliométrie ; devenir des thèses ; Monastir ;

Tunisie

Résumé - Contexte : En Tunisie, la rédaction d'une thèse est un effort de recherche qui reste peu visible pour la communauté scientifique. Objectifs : Dresser le profil bibliométrique et le devenir des thèses soutenues à la faculté de médecine de Monastir. Matériel et méthodes : Étude bibliométrique transversale menée sur l'ensemble des 616 thèses soutenues à la faculté de médecine de Monastir de 2001 à 2005. Résultats : Les disciplines médicale et chirurgicale ont été les plus pourvoyeuses de sujets de thèses (respectivement avec $45 \%$ et $40 \%$ ); 55,5\% de thèses ont été dirigées par des assistants hospitalo-universitaires. La productivité globale moyenne était de 0,6 thèse/enseignant/année. Les études cliniques représentaient la majorité des thèmes $(66,6 \%)$. Le résumé était absent dans $43 \%$ des thèses. Parmi les mots-clés cités, au moins un n'était pas un mot MeSH dans 39,3\%. Quatre-vingt-huit articles issus de thèses $(14,3 \%)$ ont été publiés dans des revues indexées avec une différence statistiquement significative en fonction des disciplines $\left(p<10^{-3}\right)$ et le type d'étude $(p=0,004)$. La médecine préventive et communautaire avait le meilleur indice de devenir des thèses, calculé en tenant compte du nombre d'enseignants par spécialité, du nombre de thèses produites et d'articles issus de ces thèses. Conclusion : Le faible taux de publications nous incite à réfléchir sur la valeur pédagogique de cet effort.
\end{abstract}

Keywords:

Bibliometrics;

future of dissertations;

Monastir;

Tunisia

Abstract - Background: In Tunisia, writing a dissertation is a research effort that goes largely unnoticed in the scientific community. Objectives: To develop the bibliometric profile and determine the future of dissertations defended in the faculty of medicine in Monastir. Methods: The bibliometric transversal study was conducted on 616 dissertations defended in the faculty of medicine in Monastir between 2001 and 2005. Results: The majority of dissertation topics come from the medical and surgical fields at a respective rate of $45 \%$ and $40 \%$. University hospital assistants led $55.5 \%$ of dissertations. The productivity average was 0.6 dissertation/teacher/year. Clinical studies accounted for the majority of topics $(66.6 \%)$. No abstract was provided in $43 \%$ of cases. In $39.3 \%$ of cases, at least one of the listed keywords was not among the MeSH terms. Some 88 articles resulting from dissertations $(14.3 \%)$ were published in indexed journals, with a statistically significant difference between disciplines $\left(p<10^{-3}\right)$ and the type of study $(p=0.004)$. The department of community and preventive 
medicine showed the best prospects for the future of dissertations, an estimation that took into account the number of teachers per department, the number of dissertations and articles resulting from these dissertations. Conclusion: The low publication rate prompts us to reconsider the pedagogical value of these efforts.

\section{Introduction}

La publication scientifique est une des missions des facultés de médecine. Leur rayonnement et leur notoriété dépendent aussi bien de la qualité de leur enseignement que de leur production scientifique ${ }^{[1]}$.

Par ailleurs, les thèses pour le diplôme d'état de doctorat en médecine constituent un relais important de production de connaissance ${ }^{[2]}$. Elles représentent un investissement en effort et en temps, aussi bien pour l'étudiant que son directeur enseignant. En Tunisie, l'élaboration et la soutenance d'une thèse sont obligatoires pour être diplômé et pour pouvoir exercer la médecine. Cependant cet effort de recherche reste peu visible pour la communauté scientifique. Avec le développement de la scientométrie, nouvelle discipline basée sur la bibliométrie $^{[3]}$, la réflexion sur le résultat de cet effort est devenue impérative.

L'objectif de ce travail est de dresser le profil bibliométrique des thèses soutenues à la faculté de médecine de Monastir (FMM) entre 2001 et 2005, d'étudier les résultats de leur éventuelle valorisation scientifique et de déterminer les facteurs associés à leur publication dans des revues indexées.

\section{Matériel et méthodes}

Stratégies générales

Il s'agit d'une étude bibliométrique descriptive transversale de l'ensemble des thèses pour le diplôme d'état de doctorat en médecine soutenues à la FMM au cours d'une période de cinq ans, entre le $1^{\text {er }}$ janvier 2001 et le 31 décembre 2005.

Au milieu de la période d'étude (2003), 178 enseignants contribuaient à la formation des étudiants de la FMM : 29 professeurs hospitalo-universitaires,
10 professeurs universitaires, 50 maîtres de conférences agrégés, 3 maîtres de conférences, 79 assistants hospitalo-universitaires et 7 maîtres assistants. Par ailleurs, des enseignants d'autres facultés de médecine de Tunisie peuvent diriger des thèses qui seront soutenues par des étudiants inscrits dans la FMM.

Les données ont été collectées à travers la consultation directe de chaque thèse dans la bibliothèque de la faculté. La page de couverture ainsi que le résumé, ou à défaut la conclusion de chaque thèse, ont fait l'objet d'une lecture directe, systématique et minutieuse.

Un complément d'information sur les mentions attribuées par le jury a été recueilli à travers le registre des thèses, tenu par le service de scolarité. Le jury peut attribuer une de ces quatre mentions : « honorable », « très honorable », « très honorable avec félicitation du jury » ou «proposition au prix de thèse ».

Le devenir de la thèse, en termes de valorisation scientifique, a été étudié à travers une recherche documentaire dans les trois bases de données «Medline », «Scopus » et «article@INIST» ainsi qu'à l'aide du moteur de recherche scientifique «Scholar Google ». Une requête combinant les noms des auteurs potentiels de la publication (étudiant et/ou directeur(s) de la dissertation) a permis de vérifier l'éventuelle publication issue de la thèse.

De plus, tous les mots-clés cités dans toutes les thèses ont été recherchés mot à mot sur Medline à l'aide du moteur de recherche HONsélect (en langue française).

L'ensemble des données présentes ont été recueillies à l'aide d'une grille standardisée décrivant l'auteur, le(s) directeur(s) de la dissertation, les membres du jury, les mots-clés, le type d'étude et la qualité rédactionnelle de la thèse. 


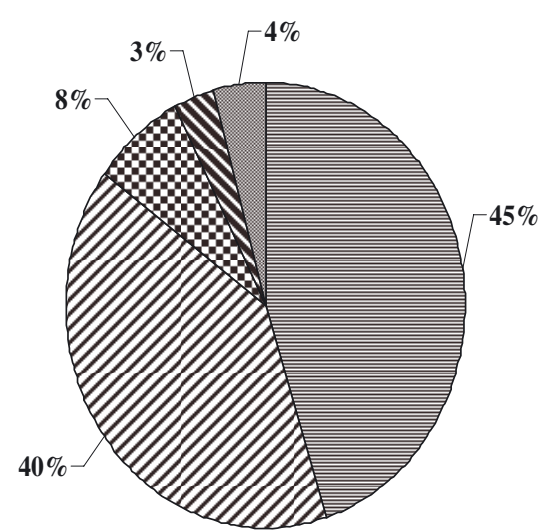

目 Médicale

$\square$ Chirurgicale

国 Fondamentale/Biologique/Mixte

s Santé Publique

D Divers

Fig. 1. Distribution des thèses soutenues à la Faculté de médecine de Monastir de 2001 à 2005, selon la discipline.

Les données ont été saisies et analysées en utilisant un matériel informatique. En plus de l'étude descriptive, les tests statistiques appropriés des déterminants d'un travail de thèse publié ont été utilisés au seuil de signification de $5 \%$.

\section{Définitions opérationnelles}

Disposant de la répartition des enseignants de la FMM en fonction de la spécialité, nous avons calculé deux indicateurs afin d'évaluer la productivité des différentes spécialités en termes de thèses et de publications qui en sont issues. Nous avons considéré l'année 2003 comme année centrale de la période de l'étude.

- Ratio thèse-enseignant : c'est le nombre de thèses réalisées au sein d'une spécialité donnée, pendant la durée d'étude (cinq ans), rapporté au nombre moyen d'enseignants qui appartenaient à cette spécialité.

- L'indice de devenir des thèses : c'est le produit du nombre d'articles publiés issus de thèses et le ratio thèse-enseignant de la même spécialité pendant toute la période de l'étude.

\section{Résultats}

Caractéristiques bibliométriques des thèses étudiées

Nous avons recensé 616 thèses soutenues à la FMM sur une période de cinq ans, soit une moyenne de
123 thèses par année. Le maximum de thèses a été soutenu au cours de l'année 2003 (133 thèses) et le minimum en 2001 (115 thèses).

L'âge moyen des candidats au moment de la soutenance de leur thèse était $29,3 \pm 2,42$ ans avec des extrêmes allant de 25 ans à 40 ans. Le taux global des thésards de genre féminin pendant cette période de cinq ans était de 44,2\%. Cette proportion est passée de $39 \%$ en 2001 à $51 \%$ en 2005.

La mention attribuée par le jury a été retrouvée pour 437 thèses. Elle était « très honorable avec félicitation du jury » dans $78 \%$ des thèses et 78 dissertations ont été proposées au prix de thèse.

Cinq cent vingt quatre thèses (soit $85 \%$ ) ont été réalisées dans les deux disciplines médicale (277) et chirurgicale (247). La proportion des thèses ayant étudié des problèmes de santé publique était la plus faible $(3,2 \%)$ (figure 1).

Les 616 thèses ont été réalisées dans 43 spécialités différentes. Le tiers d'entre elles a été produit par quatre spécialités : la pédiatrie $(10,6 \%)$, la gynécologie $(9,4 \%)$, l'orthopédie $(7,8 \%)$ et la chirurgie générale $(5,8 \%)$ (tableau I).

Les thèses ont été encadrées par un seul directeur dans 70,6\% des cas. Plus de la moitié des dissertations $(55,5 \%)$ ont été dirigées par les assistants hospitalo-universitaires de différentes spécialités. La participation des professeurs agrégés dans la direction des thèses était la plus faible $(19,2 \%)$.

Cependant, parmi les 616 thèses étudiées, 488 ont été dirigées par 162 enseignants appartenant à 
Tableau I. Répartition des thèses soutenues à la faculté de médecine de Monastir entre 2001 et 2005, selon les spécialités.

\begin{tabular}{lcc}
\hline Spécialités & Nombre de thèses & Proportion des thèses (\%) \\
\hline Pédiatrie & 65 & 10,6 \\
\hline Gynécologie obstétrique & 58 & 9,4 \\
\hline Orthopédie & 48 & 7,8 \\
\hline Chirurgie générale & 36 & 5,8 \\
\hline Cardiologie & 27 & 4,4 \\
\hline Chirurgie pédiatrique & 26 & 4,2 \\
\hline Psychiatrie & 25 & 4,1 \\
\hline Radiologie & 24 & 3,9 \\
\hline Ophtalmologie & 22 & 3,6 \\
\hline Neurochirurgie & 20 & 3,2 \\
\hline Réanimation médicale & 20 & 3,2 \\
\hline Néphrologie & 18 & 2,9 \\
\hline Pneumologie & 18 & 2,9 \\
\hline ORL & 18 & 2,9 \\
\hline Dermatologie & 17 & 2,8 \\
\hline Médecine communautaire & 16 & 2,6 \\
\hline Anesthésie réanimation & 16 & 2,6 \\
\hline Anatomie pathologique & 16 & 2,6 \\
\hline Rhumatologie & 15 & 2,4 \\
\hline Autres spécialités & 111 & 18 \\
\hline Total & $\mathbf{6 1 6}$ & $\mathbf{1 0 0}$ \\
\hline & & \\
\hline & 16 & \\
\hline
\end{tabular}

la FMM. Ainsi, la productivité globale moyenne à la faculté était de trois thèses par enseignant durant les cinq années de l'étude, soit 0,6 thèse par enseignant et par année, avec des variations entre 0,2 thèse/enseignant/an et 1,7 thèse/enseignant/an pour certaines spécialités (tableau II).

Les travaux de recherche originaux prédominaient $(92,4 \%)$ et $6 \%$ étaient de nature pédagogique (aide à l'enseignement).

La majorité des thèses traitait des sujets d'ordre clinique $(66,6 \%)$ et épidémiologique $(26,1 \%)$.

Parmi les études cliniques, 81,2\% étaient des études de cas (333 thèses). Cent vingt trois études épidémiologiques étaient d'ordre descriptif (tableau III).
Concernant la qualité rédactionnelle, toutes les thèses ont été rédigées en langue française. Le titre de la thèse était informatif dans $82 \%$ d'entre elles. Il était court et précis dans $93 \%$ des thèses. Parmi les mots-clés cités, au moins un n'était pas un mot MeSH dans 39,3 \% des thèses.

Le résumé était présent dans $57 \%$ des dissertations, respectant la structure IMRAD dans $84 \%$ d'entre elles. L'objectif de l'étude a été clairement exposé dans 80,8\% des résumés (ou à défaut la conclusion). La population cible a été mal décrite et le type d'étude n'était pas clairement mentionné dans $4,2 \%$ et $6 \%$ des parties consultées respectivement. Les principaux résultats étaient présents et bien exposés et la conclusion était concordante 
Tableau II. Répartition du ratio thèse-enseignant de la faculté de médecine de Monastir en fonction des spécialités entre 2001 et 2005 .

\begin{tabular}{|lcccc}
\hline Spécialités & $\begin{array}{c}\text { Nombre } \\
\text { de thèses }\end{array}$ & $\begin{array}{c}\text { Nombre des } \\
\text { enseignants }\end{array}$ & $\begin{array}{c}\text { Ratio thèse- } \\
\text { enseignant }\end{array}$ & $\begin{array}{c}\text { Ratio thèse- } \\
\text { enseignant annuel }\end{array}$ \\
\hline Neurochirurgie & 17 & 2 & 8,5 & 1,7 \\
Médecine de travail & 11 & 2 & 5,5 & 1,1 \\
\hline Médecine communautaire & 15 & 3 & 5 & 1 \\
\hline Orthopédie & 34 & 7 & 4,85 & 0,97 \\
\hline Pédiatrie & 62 & 14 & 4,43 & 0,88 \\
\hline Chirurgie pédiatrique & 24 & 6 & 4 & 0,8 \\
\hline Pneumologie & 12 & 3 & 4 & 0,8 \\
\hline Gynécologie obstétrique & 41 & 11 & 3,72 & 0,74 \\
\hline Chirurgie générale & 32 & 9 & 3,55 & 0,71 \\
\hline Médecine légale & 7 & 2 & 3,5 & 0,7 \\
\hline Chirurgie plastique et réparatrice & 7 & 2 & 3,5 & 0,7 \\
\hline Psychiatrie & 23 & 7 & 3,28 & 0,656 \\
\hline Rhumatologie & 13 & 4 & 3,25 & 0,65 \\
\hline Dermatologie & 13 & 4 & 3,25 & 0,65 \\
\hline Ophtalmologie & 19 & 6 & 3,16 & 0,632 \\
\hline Réanimation médicale & 21 & 7 & 3 & 0,6 \\
\hline Neurologie & 6 & 2 & 3 & 0,6 \\
\hline Médecine interne+endocrinologie & 13 & 5 & 2,6 & 0,52 \\
\hline Néphrologie & 17 & 7 & 2,43 & 0,48 \\
\hline Cardiologie & 19 & 8 & 2,375 & 0,475 \\
\hline Maladie infectieuse & 9 & 4 & 2,25 & 0,45 \\
\hline Anatomie pathologique & 9 & 4 & 2,25 & 0,45 \\
\hline ORL & 18 & 8 & 2,25 & 0,45 \\
\hline Urologie & 4 & 2 & 2 & 0,4 \\
\hline Radiologie & 19 & 10 & 1,9 & 0,38 \\
\hline Autres spécialités & 23 & $\mathbf{1 6 2}$ & $\mathbf{3 , 0 1}$ & 0,2 \\
\hline Total & $\mathbf{4 8 8}$ & & $\mathbf{0 , 6}$ \\
\hline
\end{tabular}

avec les résultats présentés dans $95,7 \%$ des parties consultées.

\section{Devenir des thèses étudiées}

Parmi les 616 thèses soutenues à la FMM de 2001 à 2005, $88(14,3 \%)$ ont servi de support à des publications dans des revues indexées. La majorité des articles a été publiée en 2005 (21) et en 2003 (14). Tous les thèmes publiés étaient issus des thèses de nature originale. La langue française était la langue de rédaction des $4 / 5^{\mathrm{e}}$ des articles.

Au total 54 journaux ont publié les 88 articles issus de thèses. Quatre-vingt-trois $(94,4 \%)$ ont été publiés dans un seul journal et cinq $(5,6 \%)$ ont été publiés dans deux journaux différents.

La «Tunisie Médicale » venait en tête des journaux sollicités par les auteurs; 17 articles issus des thèses étudiées y ont été publiés. Les revues «l'Encéphale » et «Progress in Urology» venaient en 
Tableau III. Répartition des thèses soutenues à la faculté de médecine de Monastir entre 2001 et 2005, selon le type d'étude.

\begin{tabular}{llll}
\hline Type d'étude & \multicolumn{1}{c}{$\begin{array}{c}\text { Nombre de thèses }(\mathrm{n}) \\
(\boldsymbol{\%})\end{array}$} & $\begin{array}{c}\text { Nombre de thèses selon } \\
\text { les spécialités }(\boldsymbol{n}) \\
(\%)\end{array}$ \\
\hline \multirow{3}{*}{ Clinique } & étude de cas & 33381,2 & \\
& étude thérapeutique & 4711,5 & 410 \\
& étude diagnostique & 153,7 & $(66,6)$ \\
& étude pronostique & 102,4 & \\
& étude étiologique & 41 & 161 \\
Épidémiologique & 10,2 & $(26,1)$ \\
& étude économique & 12376,4 & \\
& descriptive & 2616,1 & 9 \\
analytique & 116,8 & $(1,5)$ \\
Science fondamentale & évaluative & 10,6 & 36 \\
Autres & écologique & 111,1 & $(5,8)$ \\
\hline
\end{tabular}

deuxième position avec cinq articles pour chacun des deux journaux. Plus de la moitié des articles (58 \%) était issue de thèses dirigées par un assistant hospitalo-universitaire. Le nom de l'étudiant auteur a été annoncé dans $55 \%$ des publications. Il a été cité dans la plus part des cas $(77,1 \%)$ en deuxième, troisième ou quatrième position et n'était au premier rang que dans 4,2 \% des cas. Par ailleurs, parmi les 78 thèses qui ont été proposées au prix de thèse, huit seulement ont fait l'objet d'une publication scientifique.

La répartition des sujets de thèses publiés dans des revues indexées, selon la discipline, montre que $51,1 \%$ provenaient des différentes spécialités médicales. Les problèmes de santé publique et les études fondamentales ont représenté $20,5 \%$ des articles publiés issus des travaux de thèses.

Sur l'ensemble des 616 thèses dirigées par des enseignants de la FMM ou d'autres facultés de médecine, cinq spécialités sont parvenues à publier plus de la moitié d'articles issus de thèses (48 articles), à savoir la psychiatrie $(n=9)$, la médecine préventive et communautaire $(n=8)$, la réanimation médicale $(n=8)$, la pédiatrie $(n=8)$ et la chirurgie pédiatrique $(n=7)$.

Or, vu que la contribution des différentes spécialités aux publications ne dépend pas uniquement du nombre d'articles mais également du nombre total des thèses réalisées ajustées sur le nombre moyen des enseignants de cette spécialité, nous avons calculé l'indice de devenir des thèses dirigées par les enseignants appartenant à la FMM. Ainsi, la médecine préventive et communautaire avait le meilleur indice de devenir des thèses (35), suivi, par la psychiatrie $(29,52)$ et la chirurgie pédiatrique $(28)$ (tableau IV).

Par ailleurs, l'étude des déterminants de publication d'articles issus de thèses a fait ressortir une association statistiquement significative avec la nature de la discipline et le type d'études. En effet, la moitié des thèses soutenues en santé publique était à l'origine d'au moins une publication $\left(p<10^{-3}\right)$ et $21,1 \%$ des thèses de type épidémiologique donnaient issue à des publications contre $12,7 \%$ pour les thèses de type clinique $(p=0,004)$. Par ailleurs, la publication était indépendante du grade du premier directeur ( $p=0,345)$, de la mention de la thèse 
Tableau IV. Répartition des spécialités selon leur indice de devenir de thèses dirigées par des enseignant de la faculté de médecine de Monastir entre 2001 et 2005.

\begin{tabular}{|llll}
\hline Spécialités & $\begin{array}{l}\text { Ratio thèse- } \\
\text { enseignant } \\
(\mathbf{5} \text { années })\end{array}$ & $\begin{array}{l}\text { Nombre de } \\
\text { de thèses publiées }\end{array}$ & $\begin{array}{l}\text { Indice de devenir } \\
\text { des thèses }\end{array}$ \\
\hline Médecine communautaire & 5 & 7 & 35 \\
\hline Psychiatrie & 3,28 & 9 & 29,52 \\
\hline Chirurgie pédiatrique & 4 & 7 & 28 \\
\hline Pédiatrie & 4,43 & 6 & 26,58 \\
\hline Réanimation médicale & 3 & 8 & 24 \\
\hline Rhumatologie & 3,25 & 5 & 16,25 \\
\hline Pneumologie & 4 & 4 & 16 \\
\hline Ophtalmologie & 3,16 & 4 & 12,64 \\
\hline Cardiologie & 2,375 & 4 & 9,5 \\
\hline Chirurgie générale & 3,55 & 2 & 7,1 \\
\hline Dermatologie & 3,25 & 2 & 6,5 \\
\hline Médecine de travail & 5,5 & 1 & 5,5 \\
\hline Médecine interne+endocrinologie & 2,6 & 2 & 5,2 \\
\hline Gynécologie obstétrique & 3,72 & 1 & 3,72 \\
\hline Chirurgie plastique et réparatrice & 3,5 & 1 & 3,5 \\
\hline gastrologie & 1,5 & 2 & 3 \\
\hline Anesthésie-réanimation & 1,5 & 2 & 3 \\
\hline Maladie infectieuse & 2,25 & 1 & 2,25 \\
\hline Urologie & 2 & 1 & 2 \\
\hline Biophysique & 0,5 & 1 & 0,5 \\
\hline
\end{tabular}

$(p=0,688)$ et du genre du thésard $(p=0,466)$ (tableau V).

\section{Discussion}

Dans notre travail, l'étude bibliométrique des thèses a été basée sur la construction d'une grille de collecte des informations, ne tenant compte que de certaines parties de la thèse (page de garde et résumé ou à défaut la conclusion). Ces parties peuvent occulter des éléments nécessaires à l'analyse de la qualité de la thèse. Malgré ce biais, les résultats rapportés reflètent la partie émergée de l'iceberg « qualité rédactionnelle ».

En outre, le devenir de la thèse a été étudié à travers une recherche documentaire dans les trois bases de données «Medline », «Scopus» et «article@INIST» ainsi qu'à l'aide du moteur de recherche scientifique «Scholar Google ». L'interrogation des ces bases de données bibliographiques, associée au recul minimum de cinq ans que nous avons fixé, rendent peu probable la non visibilité d'un article publié.

Notre étude a montré que $85 \%$ des thèses étudiées ont été réalisées dans les deux disciplines médicale et chirurgicale avec une légère prédominance pour les spécialités médicales (45\% vs. $40 \%$ ). En effet, le choix du sujet de thèse est principalement orienté en fonction du cursus des études et plus précisément en fonction des stages de l'internat.

Dans une étude menée sur un échantillon aléatoire de 300 thèses présentées dans les 36 universités médicales françaises, de 1993 à 1997, 79,3 \% des thèses provenaient des services médicaux ${ }^{[4]}$. La prédominance des spécialités médicales et chirurgicales comme sujets de thèses a été également 
Tableau V. Facteurs déterminants des publications issues des sujets de thèses étudiés.

\begin{tabular}{|c|c|c|c|c|c|}
\hline Variables & & $\begin{array}{l}\text { Nombre de } \\
\text { thèses } \\
\text { publiées }\end{array}$ & $\begin{array}{l}\text { Nombre de } \\
\text { thèses non } \\
\text { publiées }\end{array}$ & $\begin{array}{l}\text { Proportion de } \\
\text { thèses } \\
\text { publiées }(\%)\end{array}$ & $\begin{array}{l}\text { Chi } 2 \\
(p)\end{array}$ \\
\hline \multirow{3}{*}{ Type d'étude } & Clinique & 52 & 358 & 12,68 & \multirow{3}{*}{$\begin{array}{l}13,46 \\
(0,004)\end{array}$} \\
\hline & Épidémiologique & 34 & 127 & 21,11 & \\
\hline & Science fondamentale & 2 & 7 & 22,2 & \\
\hline \multirow{5}{*}{ Disciplines } & Santé publique & 10 & 10 & 50 & \multirow{5}{*}{$\begin{array}{l}28,36 \\
\left(<10^{-3}\right)\end{array}$} \\
\hline & Fondamentale & 8 & 39 & 17 & \\
\hline & Médicale & 45 & 232 & 16,2 & \\
\hline & Chirurgicale & 24 & 223 & 9,7 & \\
\hline & Autres & 1 & 25 & 3,8 & \\
\hline \multirow{3}{*}{$\begin{array}{l}\text { Grade du directeur } \\
\text { de thèse }\end{array}$} & Professeur & 25 & 131 & 16 & \multirow{3}{*}{$\begin{array}{l}2,128 \\
(0,345)\end{array}$} \\
\hline & $\begin{array}{l}\text { Maître de } \\
\text { conférences }\end{array}$ & 12 & 106 & 10,2 & \\
\hline & Assistant & 51 & 291 & 15 & \\
\hline \multirow{3}{*}{$\begin{array}{l}\text { Mention de } \\
\text { la thèse }\end{array}$} & $\begin{array}{l}\text { Proposition au prix } \\
\text { de thèse }\end{array}$ & 8 & 70 & 10,3 & \multirow{3}{*}{$\begin{array}{l}1,476 \\
(0,688)\end{array}$} \\
\hline & $\begin{array}{l}\text { Très honorable avec } \\
\text { félicitation du jury }\end{array}$ & 46 & 295 & 13,5 & \\
\hline & Très honorable & 1 & 16 & 5,9 & \\
\hline \multirow{2}{*}{ Genre du thésard } & Masculin & 46 & 298 & 13,4 & \multirow{2}{*}{$\begin{array}{l}0,531 \\
(0,466)\end{array}$} \\
\hline & Féminin & 42 & 230 & 15,4 & \\
\hline
\end{tabular}

rapportée dans une étude togolaise portant sur le devenir scientifique des thèses de médecine soutenues à l'université de Lomé au cours de la période de 1993-2002 ${ }^{[1]}$.

Par ailleurs, dans notre travail, le tiers des thèses a été produit par quatre spécialités, à savoir la pédiatrie, la gynécologie, l'orthopédie et la chirurgie générale.

Dans une étude française descriptive des thèses de médecine (942) ${ }^{[5]}$, les spécialités les plus pourvoyeuses de sujets de thèses étaient, par ordre décroissant : les maladies infectieuses, la gériatrie, la médecine générale, la pédiatrie, la cardiologie, la cancérologie, la neurologie, la gynécologie et l'orthopédie. La primauté des maladies infectieuses suivie par la cancérologie est retrouvée dans l'analyse des thèses d'exercice soutenues par les internes en médecine générale de la faculté de médecine
Paris Descartes de janvier 2005 à décembre 2007 (259 thèses) ${ }^{[6]}$.

La différence remarquée entre les spécialités les plus productives de thèses dans notre faculté et celles sus citées peut être expliquée par le niveau du développement des spécialités médicales ou chirurgicales entre ces facultés ainsi que le nombre des services et d'enseignants pour chaque spécialité.

Par ailleurs, le ratio thèse-enseignant nous a permis d'identifier les spécialités les plus productives de thèses, durant la période étudiée. En effet, la productivité globale moyenne à la FMM, de 2001 à 2005, était de trois thèses par enseignant durant cinq années ; soit 0,6 thèse/enseignant/année. Ce ratio annuel a varié entre 0,2 et 1,7 . La productivité globale moyenne à la faculté de médecine de Sousse (Tunisie) de 1981 à 2000 fut de 0,78 thèse par enseignant et par année ${ }^{[7]}$. 
La différence observée pour ce ratio entre les différents services et surtout son ampleur sont difficilement explicables et mériteraient par conséquent des études complémentaires pour être expliquées.

Nous avons trouvé que les études d'ordre clinique et épidémiologiques étaient les plus fréquentes, au détriment des études portant sur des sciences fondamentales. Cette constatation a été rapportée dans de nombreuses études ${ }^{[1,4]}$. En effet, la faible fréquence des études portant sur des sciences fondamentales peut s'expliquer par le fait que ces études nécessitent parfois la réalisation d'examens paracliniques très spécialisés et onéreux.

Dans notre travail, nous avons noté l'absence du résumé dans $43 \%$ des thèses. Ceci est dû au caractère non obligatoire du résumé comme une partie individualisée de tout travail avant 2004. L'absence des résumés de thèses a été rapportée aussi dans la littérature. En effet, $34 \%$ des thèses soutenues à la faculté de médecine de Tours (France) de 1989 à 1992 ne comportaient pas de résumé et ce dernier apparaissait souvent peu informatif ${ }^{[8]}$.

Il en découle que le résumé, souvent considéré comme un miroir du travail, ne remplira pas sa fonction de valorisation de la thèse et participera mal à sa bonne diffusion en l'absence d'une rigueur dans sa rédaction.

Parmi les 616 thèses que nous avons étudiées, seules $88(14,3 \%)$ ont fait l'objet de publications indexées dans les bases consultées, soit une thèse sur sept. Cette étude est, à notre connaissance, la première du genre en Tunisie qui a cherché à évaluer le devenir scientifique des thèses de médecine et leur impact dans la production scientifique des enseignants chercheurs. Cette faible proportion de thèses publiées a été rapportée dans plusieurs études. Foucheyrand ${ }^{[8]}$ a trouvé dans son travail que 30,3\% de thèses ont servi de support pour des publications mais seule 8,9\% étaient publiés dans des revues indexées. Salmi et al. ${ }^{[4]}$, qui ont utilisé le nom du candidat et/ou du directeur pour leur requête sur Medline, ont mentionné que $17 \%$ des thèses ont donné lieu à une publication, ce qui les conduit à se poser la question sur la valeur éducative des thèses en médecine. De même, 54 (22,5\%) articles issus de thèses soutenues à la faculté de médecine de Lomé (Togo) ont été publiées dans des revues indexées ${ }^{[1]}$.

Dans notre étude, nous avons constaté que les 78 dissertations proposées pour prix de thèse avaient un faible taux de publication $(10,3 \%)$. Ceci nous incite à réfléchir sur les critères d'attribution de la mention par le jury.

À l'opposé, le faible taux de publication observé dans notre travail contraste avec celui rapporté dans une étude rétrospective menée sur 160 thèses soutenues dans une université médicale de Delhi en Inde ${ }^{[9]}$, pendant la même période que notre étude (2001-2005). En effet, le taux de publication en journaux répertoriés des thèses universitaires était de $30 \%$. De même, une proportion de publications plus importante que celle observée pour nos thèses est également rapportée dans une étude bibliométrique de toutes les thèses de chirurgie dentaire soutenues au service d'orthopédie dento-faciale de l'Université de Dakar (Sénégal) entre 1991 et $2008^{[10]}$. Parmi ces thèses $35,7 \%$ ont fait l'objet de publications indexées.

Par ailleurs, dans notre travail, l'étude des facteurs déterminants de la publication d'une thèse a montré que la moitié des thèses soutenues en santé publique était à l'origine d'au moins une publication et que $21,1 \%$ des thèses de type épidémiologique donnent issue à des publications contre $12,7 \%$ pour les thèses cliniques. Par contre, cette étude analytique n'a pas montré de différence statistiquement significative entre les grades des directeurs, entre les deux genres des thésards et en fonction de la mention de la thèse.

Vogel et Windeler ${ }^{[11]}$ ont évalué les facteurs qui ont influencé la publication d'une thèse à travers l'analyse multivariée de 371 thèses de médecine. Ils ont montré que trois facteurs essentiels influençaient la publication des thèses : un résultat positif de l'étude, un encadreur de thèse habitué à publier des articles et la qualité de l'analyse statistique des données de la thèse.

À l'inverse, Vickers et Smith ${ }^{[12]}$ ont cherché les causes du non publication des thèses et ont conclu 
que la cause principale était la pauvreté du chapitre matériel et méthodes.

\section{Conclusion}

Notre étude peut être le point de départ d'une réflexion pédagogique sur le devenir des thèses élaborées et soutenues dans notre faculté. En effet, la thèse pour le diplôme d'état de doctorat en médecine constitue un travail original marquant la fin d'un long cycle d'études et l'occasion d'un effort de réflexion, d'écriture et de synthèse. Cependant, Il ne suffit pas de faire une bonne thèse pour être considéré comme un bon chercheur et il faut aussi publier des articles avec une audience nationale et internationale. Ainsi, la valorisation de la thèse est impérative. Pour atteindre cet objectif, il est nécessaire de développer l'enseignement de la rédaction médicale, de la lecture critique d'articles scientifiques, ainsi que des outils de méthodologie statistique dans la formation de base des études médicales au sein de nos facultés de médecine, tout en profitant du développement moderne des nouvelles technologies de communication et d'information.

\section{Contributions}

Kamel Ben Salem et Ahmed Ben Abdelaziz ont élaboré le protocole de recherche et la grille de collecte de données; Nizar Mosbahi a participé au recueil des données; Ines Bouanene et Sana Elmhamdi ont procédé à l'analyse statistique et à l'interprétation des résultats; Kamel Ben Salem et Ines Bouanene ont pris en charge l'écriture du manuscrit.

\section{Remerciements}

Notre travail rentre dans le cadre d'une réflexion sur l'optimalisation de la dissertation en facilitant son évolution en un article scientifique publiable ou publié. Il fait partie d'un travail de recherche multicentrique sur le devenir des thèses à l'échelle nationale, mené par le Groupe de Recherche Inter Facultaire sur le Devenir des Dissertations dans le domaine de la santé (GRIF2D).

\section{Références}

1. Pitche P, Onipoh D, Tchangai-Walla K. Devenir scientifique des thèses pour le diplôme d'état de doctorat en médecine soutenues à l'université de Lomé (Togo). Pédagogie Médicale 2007 ; 8 :24-9.

2. Nougairede P. Étude Publiothèse : taux d'exploitation des thèses en médecine générale au sein de la faculté Denis Diderot Paris 7. Thèse pour le doctorat en médecine 2009.

3. Lauri P. La Bibliométrie, un indicateur de tendance. Int $\mathbf{J}$ Inform Sci Decis Making 1997;1:28-36.

4. Salmi LR, Gana S, Mouillet E. Publication pattern of medical theses, France, 1993-98. Med Educ 2001 ;35 :18-21.

5. Remacle A. La thèse en médecine générale : étude descriptive des thèses de médecine générale soutenues à l'université Bordeaux 2-Victor Segalen de 1995 à 2000, Thèse. Université de médecine Bordeaux II ; 2002.

6. Chevalier M. Analyse des thèses d'exercice soutenues par les internes en médecine générale de la Faculté de Médecine Paris Descartes de janvier 2005 décembre 2007. Thèse de médecine, Faculté de Médecine Paris Descartes (Paris), 23 juin 2009.

7. Laribi L. Étude bibliométrique des thèses soutenues à la Faculté de Médecine de Sousse de 1981 à 2000. Thèse de médecine, Faculté de Médecine «Ibn El Jazzar » de Sousse, 30 juin 2004.

8. Foucheyrand P. Analyse descriptive, méthodologique et devenir des thèses en médecine. Thèse de médecine, Faculté de médecine de Tours, 1994.

9. Dhaliwal U, Singh N, Bhatia A. Masters theses from a university medical college : publication in indexed scientific journals. Indian J Ophthalmol $2010 ; 58(2): 101-4$.

10. Mbasse N. Bibliométrie et devenir des thèses soutenues dans le service d'orthopédie dento-faciale de Dakar de 1991 a 2008. Thèse en Chirurgie Dentaire. Faculté d'odonto-stomatologie. Université Cheikh Anta DIOP de Dakar (Sénégal), 10 juillet 2009.

11. Vogel U, Windeler J. Factors modifying frequency of publications of clinical research results exemplified by medical dissertations. Dtsch Med Wochenschr $2000 ; 125: 110-3$.

12. Vickers AJ, Smith C. Incorporating data from dissertations in systematic reviews. Int J Technol Assess Health Care $2000 ; 16: 711-3$

Correspondance et offprints : Kamel Ben Salem, Département de Médecine Communautaire, Faculté de Médecine de Monastir 5019 Monastir, Tunisie.

Mailto : Kamel.BenSalem@gmail.com 